\title{
Magnetic Imaging of Pearl Vortices in Artificially Layered $\left(\mathrm{Ba}_{0.9} \mathrm{Nd}_{0.1} \mathrm{CuO}_{2+x}\right)_{m} /\left(\mathrm{CaCuO}_{2}\right)_{n}$ Systems
}

\author{
F. Tafuri, ${ }^{1,2}$ J. R. Kirtley, ${ }^{2}$ P. G. Medaglia, ${ }^{3}$ P. Orgiani, ${ }^{3}$ and G. Balestrino ${ }^{3}$ \\ ${ }^{1}$ INFM-Coherentia, Dipartimento di Ingegneria dell'Informazione, Seconda Università di Napoli, Aversa (CE), Italy \\ ${ }^{2}$ IBM Watson Research Center, Route 134 Yorktown Heights, New York, USA \\ ${ }^{3}$ INFM-Coherentia, Dipartimento di Ingegneria Meccanica, Università di Roma Tor Vergata, Roma, Italy
} (Received 17 June 2003; published 16 April 2004)

We have used scanning SQUID magnetometry to image vortices in ultrathin $\left(\mathrm{Ba}_{0.9} \mathrm{Nd}_{0.1} \mathrm{CuO}_{2+x}\right)_{m} /$ $\left(\mathrm{CaCuO}_{2}\right)_{n}$ high temperature superconductor samples, with as few as three superconducting $\mathrm{CuO}_{2}$ planes. The Pearl lengths $\left(\Lambda=2 \lambda_{L}^{2} / d, \lambda_{L}\right.$ the London penetration depth, $d$ the superconducting film thickness) in these samples, as determined by fits to the vortex images, agree with those by local susceptibility measurements, and can be as long as $1 \mathrm{~mm}$. The in-plane penetration depths $\lambda_{a b}$ inferred from the Pearl lengths are longer than many bulk cuprates with comparable critical temperatures. We speculate on the causes of the long penetration depths, and on the possibility of exploiting the unique properties of these superconductors for basic experiments.

DOI: 10.1103/PhysRevLett.92.157006

Vortices play a central role in many aspects of superconductivity. Not only do the dynamics of vortices determine many of the transport properties of type II superconductors, especially the high critical temperature cuprates [1], but vortices are also of more general interest, since as topological defects they are of great relevance, for instance, to phase transitions [2,3]. The formation of topological defects in phase transitions has even stimulated some analogies between cosmology, gauge theories, and condensed matter physics [4,5]. Vortices in bulk type II superconductors were first predicted by Abrikosov in 1957 [6], and have since been imaged by many different experimental techniques [7]. Vortices in thin superconductors $\left(d \ll \lambda_{L}\right.$, where $d$ is the superconducting film thicknes and $\lambda_{L}$ the London penetration depth, respectively) were first described by Pearl [8] (hence "Pearl" vortices). Pearl vortices have several interesting attributes. The field strengths $h_{z}$ perpendicular to the films diverge as $1 / r$ at distances $r \ll \Lambda$ in Pearl vortices, whereas in Abrikosov vortices the fields diverge as $\ln \left(r / \lambda_{L}\right)$ [9]. Since in the Pearl vortex much of the vortex energy is associated with the fields outside of the superconductor, the interaction potential $V_{\text {int }}(r)$ between Pearl vortices has a long range component $V_{\text {int }} \sim \Lambda / r$ for $r \gg$ $\Lambda$ [8], unlike Abrikosov vortices, which have only short range interactions. The interaction between Pearl vortices $V_{\text {int }} \sim \ln (\Lambda / r)$ for $r \ll \Lambda$ leads to a BerezinskiiKosterlitz-Thouless (BKT) transition which is cut off due to screening on a scale $\Lambda$ [1]. The logarithmic interaction makes this system very similar to a Coulomb gas and ideal to study screening effects and renormalization in BKT transitions [10]. While superconducting vortices in films with thickness $d$ comparable to the London penetration depth $\lambda_{L}$ have been imaged using many techniques, to our knowledge the present work is the first to directly demonstrate experimentally the existence of
PACS numbers: 74.72.-h, 73.40.Gk, 73.40.Rw, 74.50.+r
Pearl vortices for $d \ll \Lambda$, and is also the first to use scanning susceptibility measurements to determine penetration depths in superconductors.

In the present work, two different types of $\left(\mathrm{Ba}_{0.9} \mathrm{Nd}_{0.1} \mathrm{CuO}_{2+x}\right)_{m} /\left(\mathrm{CaCuO}_{2}\right)_{n} \quad(\mathrm{CBCO})$ structures were grown: (a) the ultrathin $\left(\mathrm{Ba}_{0.9} \mathrm{Nd}_{0.1} \mathrm{CuO}_{2+x}\right)_{M} /$ $\left(\mathrm{CaCuO}_{2}\right)_{N} /\left(\mathrm{Ba}_{0.9} \mathrm{Nd}_{0.1} \mathrm{CuO}_{2+x}\right)_{M}(M / N / M)$ structure which consists of only one superconducting infinite layer (IL) block $\left(N \mathrm{CaCuO}_{2}\right.$ unit cells), sandwiched between two charge reservoir (CR) blocks ( $M$ Ba-based unit cells) and the similar $M / N / M / N / M$ structure $(M=5$ and $N=2)$; (b) the relatively thicker $\left[\left(\mathrm{Ba}_{0.9} \mathrm{Nd}_{0.1} \mathrm{CuO}_{2+x}\right)_{m} /\left(\mathrm{CaCuO}_{2}\right)_{n}\right]_{S}(m \times n$ superlattice $)$ structure which consists of $S$ sequences (with $S \geq 15$ ) of the $\left(\mathrm{Ba}_{0.9} \mathrm{Nd}_{0.1} \mathrm{CuO}_{2+x}\right)_{m} /\left(\mathrm{CaCuO}_{2}\right)_{n}$ supercells composed of $m$ Ba-based and $n$ Ca-based unit cells. All the samples were grown on (001) $\mathrm{SrTiO}_{3}$ substrates, with nominally zero miscut angle, by pulsed laser deposition (PLD), using a focused $\mathrm{KrF}$ excimer pulsed laser source $(\lambda=248 \mathrm{~nm})$ with energy areal density on the target surface of $7 \mathrm{~J} / \mathrm{cm}^{2}$ in a spot size of $2 \mathrm{~mm}^{2}$. Two sintered powder targets, with a nominal composition of $\left(\mathrm{Ba}_{0.9} \mathrm{Nd}_{0.1}\right) \mathrm{CuO}_{2}$ and $\mathrm{CaCuO}_{2}$, mounted on a multitarget system, were used. The substitution of $10 \%$ of the $\mathrm{Ba}$ atoms with trivalent $\mathrm{Nd}$ cations, even if not strictly necessary for superconductivity $[11,12]$, helped us to find the right growth conditions by slightly decreasing the uncompensation of the electrical charge in the CR block. The growth temperature was about $640^{\circ} \mathrm{C}$ and the molecular oxygen pressure was $\approx 1 \mathrm{mbar}$. At the end of the deposition procedure, an amorphous protecting layer of electrically insulating $\mathrm{CaCuO}_{2}$ was deposited on top of the film at a temperature lower than $100{ }^{\circ} \mathrm{C}$.

The SQUID microscope measurements were made at $4.2 \mathrm{~K}$ with the sample cooled and imaged in fields of a few $\mathrm{mG}$, sufficient to trap several vortices in a 
$200 \mu \mathrm{m} \times 200 \mu \mathrm{m}$ scan area. Two types of SQUID sensors were used: (1) magnetometers [13] with either square pickup loops $7.5 \mu \mathrm{m}$ on a side, or octagonal pickup loops $4 \mu \mathrm{m}$ in diameter; and (2) SQUID susceptometers [14] with a single turn field coil $20 \mu \mathrm{m}$ in diameter, with a square pickup loop $8 \mu \mathrm{m}$ across [see Fig. 2(a)].

We have performed scanning SQUID microscopy (SSM) on various $5 / 2 / 5$ monolayers, $5 / 2 / 5 / 2 / 5$ bilayers and CBCO $m \times n$ samples, and as a function of the number of the $\mathrm{CuO}_{2}$ planes. In all systems we have clearly observed Pearl vortices. This provides evidence of superconductivity complementary to traditional transport measurements [15] for the thinnest films. We show in Fig. 1 SSM images of vortices trapped in two typical samples. The thin-film limit for the two-dimensional Fourier transform of the $z$ component of the field from an isolated vortex trapped in a thin film is given by $[8,16]$ :

$$
h_{z}(k, z)=\frac{\phi_{0} e^{-k z}}{1+k \Lambda},
$$

where $z$ is the height above the film, $k=\sqrt{k_{x}^{2}+k_{y}^{2}}, \Lambda=$ $2 \lambda_{a b}^{2} / d$ is the Pearl penetration length, $\lambda_{a b}$ is the in-plane penetration depth, $d$ is the film thickness, and $\phi_{0}=$
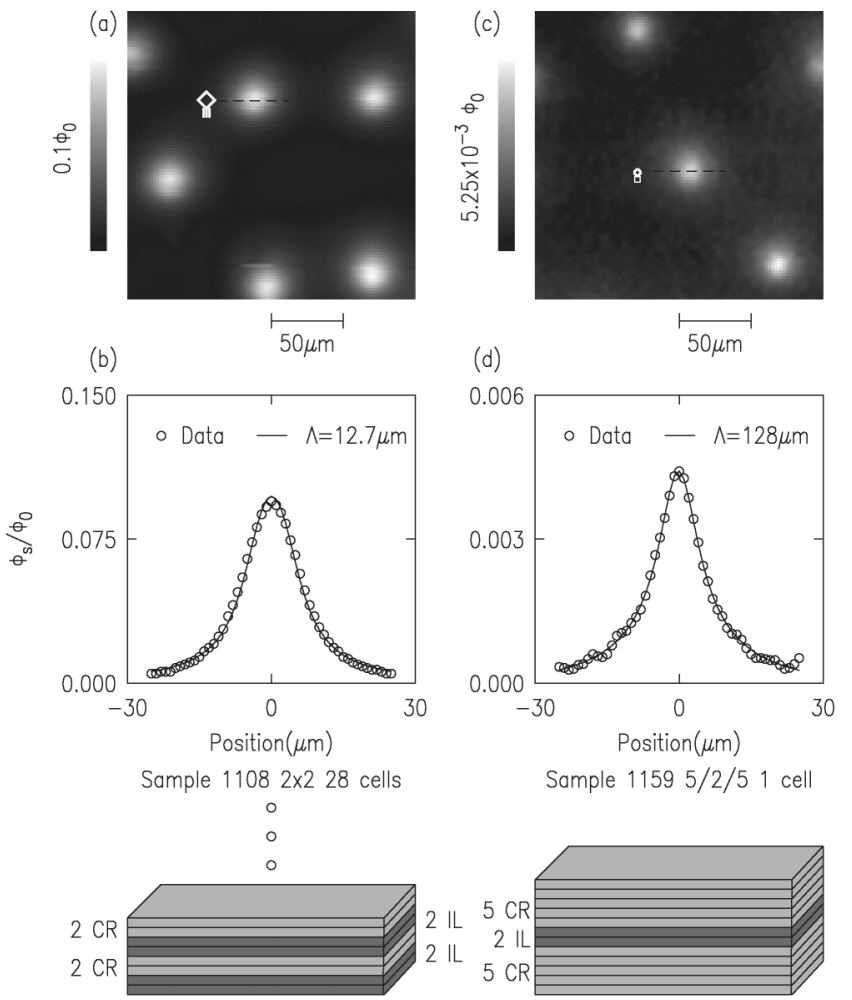

FIG. 1. SQUID microscope image and cross-sectional data [along the positions indicated by the dashed lines in (a),(c)] of vortices trapped in two CBCO samples. The SQUID pickup loops were a square $7.5 \mu \mathrm{m}$ on a side (a),(b) and an octagon $4 \mu \mathrm{m}$ on a side (c),(d). The open symbols in (b),(d) are the cross-sectional data; the solid lines in (b),(d) are fits to Eq. (1). Scaled schematics of the pickup loops used appear in (a),(c). $h c / 2 e$. We fit the data in Fig. 1 by inverting Eq. (1) to find $h(x, y, z)$, integrating the result over the known pickup loop geometry, and using $\Lambda$ as the fitting parameter. The height $z$ was determined by fitting images of vortices in $\mathrm{Nb}$ with the same SQUID magnetometer, assuming an isotropic low temperature $\mathrm{Nb}$ penetration depth of $\lambda=0.05 \mu \mathrm{m}$ [17]. We note that although the peak SQUID flux $\phi_{s}$ depends strongly on $\Lambda$, the fullwidth at half-maximum of the vortex images is relatively independent of $\Lambda$ for such thin films. For comparison, Abrikosov vortices typically couple about $0.5 \phi_{0}$ of flux into the SQUID sensor in this geometry, and are resolution limited.

The results for the Pearl lengths $\Lambda$ from such fits to images of vortices for a number of superlattice samples are summarized in Table I. The values for the film thicknesses $d$ and IL layer thickness $d_{I L}$ were obtained by assuming a thickness/layer of $3.2 \AA$ for the $\mathrm{CaCuO}_{2}$ (IL) layers, and $4.4 \AA$ for the $\mathrm{BaCuO}_{x}$ (CR) layers.

It is remarkable that Pearl vortices can be observed in films with Pearl lengths up to a millimeter long: an Abrikosov vortex in a superconductor with penetration depth $\lambda_{a b}=128 \mu \mathrm{m}$ [Fig. 1(d)] would couple a peak flux of about $1 \times 10^{-3} \phi_{0}$ into the SQUID sensor, with a peak width of about $250 \mu \mathrm{m}$, making imaging extremely difficult. However, although the peak flux from the Pearl vortex in Fig. 1(d) is relatively small $\left(\sim 4.5 \times 10^{-3} \phi_{0}\right)$, the strongly diverging fields in Pearl vortices $\left(h_{z} \sim 1 / r, r\right.$ the in-plane radius from the vortex center) give sharp peaks in the scanning SQUID image. The resulting strong contrast makes it feasible to determine the Pearl length. A consistency check on the Pearl lengths so determined can be obtained by making scanning susceptometer measurements [18]. In the measurements illustrated by Fig. 2 the sample is driven down until it comes into contact with a corner of the SQUID susceptometer, which is mounted on a flexible brass cantilever. Contact occurs at a spacing between the SQUID pickup loop and the sample surface

TABLE I. Pearl lengths $\Lambda$ of various CBCO samples. $T_{c}$ is measured by standard four-probe techniques and refers to zero resistance. We estimate uncertainties in $\Lambda$ of $\pm 20 \%$ and of $T_{c}$ of $\pm 0.25 \mathrm{~K}$.

\begin{tabular}{ccccccc}
\hline \hline Sample & Type & Cells & $d(\AA)$ & $d_{I L}(\AA)$ & $T_{c}(K)$ & $\Lambda(\mu \mathrm{m})$ \\
\hline 1159 & $5 / 2 / 5$ & 1 & 50 & 6.4 & 30 & 128 \\
1151 & $5 / 2 / 5$ & 1 & 50 & 6.4 & 35 & 205 \\
1988 & $5 / 2 / 5 / 2 / 5$ & 1 & 79 & 12.8 & 50 & 292 \\
1984 & $5 / 2 / 5 / 2 / 5$ & 1 & 79 & 12.8 & 50 & 490 \\
1987 & $5 / 2 / 5 / 2 / 5$ & 1 & 79 & 12.8 & 50 & 810 \\
1985 & $2 \times 2$ & 12 & 182 & 76.8 & 78 & 25 \\
1201 & $2 \times 2$ & 20 & 304 & 128 & 65 & 13.6 \\
1108 & $2 \times 2$ & 28 & 426 & 179.2 & 70 & 12.7 \\
1106 & $2 \times 2$ & 28 & 426 & 179.2 & 75 & 9.1 \\
1171 & $5 \times 2$ & 15 & 426 & 96 & 60 & 14.2 \\
\hline \hline
\end{tabular}


of about $5.0 \mu \mathrm{m}$, as determined by fits of similar data using a $\mathrm{Nb}$ sample, assuming a $\mathrm{Nb}$ penetration depth of $0.05 \mu \mathrm{m}$. In principle, the mutual inductance should saturate when the SQUID substrate contacts the sample. Experimentally there continues to be some change, presumably because the tilt angle between the substrate and the sample decreases.

The 2D Fourier transform of the $z$ component of the field in the pickup loop, with a current $I$ in a circular ring of radius $R$ oriented parallel to, and a height $z$ above a sample, is given by [19]

$$
h_{z}(k)=\frac{-4 \pi^{2} I R}{c} J_{1}(k R)\left(1-\frac{e^{-2 k z}}{1+k \Lambda}\right),
$$

where $J_{1}(k R)$ is a Bessel function of the first kind. The solid line in Fig. 2(b) is obtained by numerically integrating the 2D Fourier transform of Eq. (2) over the area of the pickup loop, for various values of $z$, and fit to the data by varying $\Lambda$. Figure 2(c) compares the values obtained for the Pearl lengths for a number of the CBCO samples using magnetometry and susceptometry methods. The two methods agree within experimental error over the range of Pearl lengths present [20].

As expected, the Pearl lengths are longest for the thinnest $\mathrm{CBCO}$ films. Figure 3 shows that the $\mathrm{CBCO}$ (a)

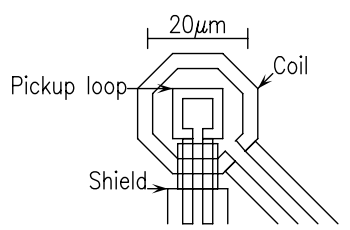

(c)

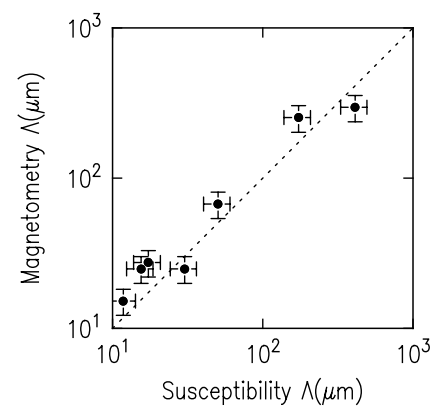

(b)
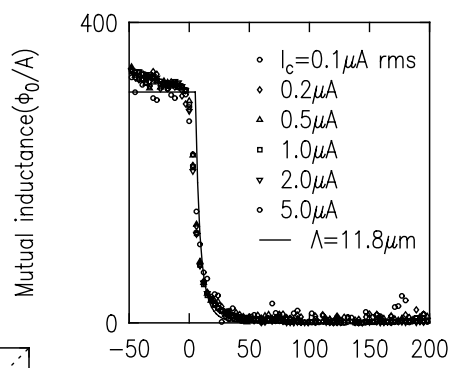

Distance from tip-sample contact $(\mu \mathrm{m})$ Sample $19852 \times 212$ cells
FIG. 2. (a) Geometry of the SQUID susceptometer used. (b) Mutual inductance between the field coil and the pickup loop, as a function of the spacing $z$ between the SQUID substrate and sample. The symbols are data, taken with various alternating currents through the field coil. The solid line is modeling using Eq. (2), with $\Lambda=11.8 \mu \mathrm{m}$. (b) Comparison of the Pearl length $\Lambda$ for a number of CBCO samples using fitting of SQUID magnetometry images of vortices (vertical axis) vs susceptibility measurements (horizontal axis). penetration depths $\lambda_{a b, h}=\sqrt{d \Lambda / 2}$ obtained assuming a homogeneous film (solid circles) are longer than for a number of hole-doped cuprates with comparable critical temperatures [21-23]. For example, optimally doped $\mathrm{YBa}_{2} \mathrm{Cu}_{3} \mathrm{O}_{7-\delta}$ (Y-123), with a $T_{c}$ of $92 \mathrm{~K}$, has $\lambda_{a b} \sim$ $0.15 \mu \mathrm{m}$ [24]. The highest $T_{c}$ CBCO sample (sample $\left.1985, T_{c}=78 \mathrm{~K}\right)$ has $\lambda_{a b, h}=0.48 \mu \mathrm{m}$. Our samples span a wide range of Pearl lengths and sheet resistances per square. Detailed measurements of the latter are given elsewhere $[11,15]$. The $2 \times 2$ superlattices have resistance per square values a factor of 10 lower than the metalinsulator limit in the $2 \times n$ superlattice series [11]. Since the mean free path in the high resistivity(but metallic, $n \sim 11$ or $5 / 2 / 5 / 2 / 5)$ films can be no shorter than the width of a $\mathrm{CuO}_{2}$ unit cell $(\sim 4 \AA)$, and since the normal state carrier sheet densities and effective masses should be similar within this series, this implies that the mean free paths in the $2 \times 2$ superlattices must be at least a few times larger than the in-plane coherence length $(\xi \sim$ $20 \AA$ ), and that the Pippard correction for the effect of a finite mean free path $l, \lambda_{\text {eff }}=\lambda_{L}(1+\xi / l)^{1 / 2}$, cannot be large. The London approximation $\left(\lambda_{L}^{2}=m^{*} c^{2} / 4 \pi n_{s} e^{2}\right.$, [25], where $m^{*}$ is the effective mass of the charge carriers) may therefore be reasonable to evaluate the superfluid density for this type of structure. If we use the standard London expression and a reasonable value of $m^{*}=5 m_{e}$ [26], we obtain $n_{s}=6.29 \times 10^{21} \mathrm{~cm}^{-3}$ for optimally doped Y-123, as compared with $n_{s}=6.28 \times$ $10^{20} \mathrm{~cm}^{-3}$ for the highest $T_{c}$ CBCO sample (1985). The corresponding areal superfluid densities per plane $n_{p}=$ $n_{s} d / N_{p}$ are $3.67 \times 10^{14} \mathrm{~cm}^{-2}$ for optimally doped Y-123 and $3.2 \times 10^{13} \mathrm{~cm}^{-2}$ for CBCO sample $1985\left(N_{p}\right.$ is the number of superconducting $\mathrm{CuO}_{2}$ planes). The superfluid

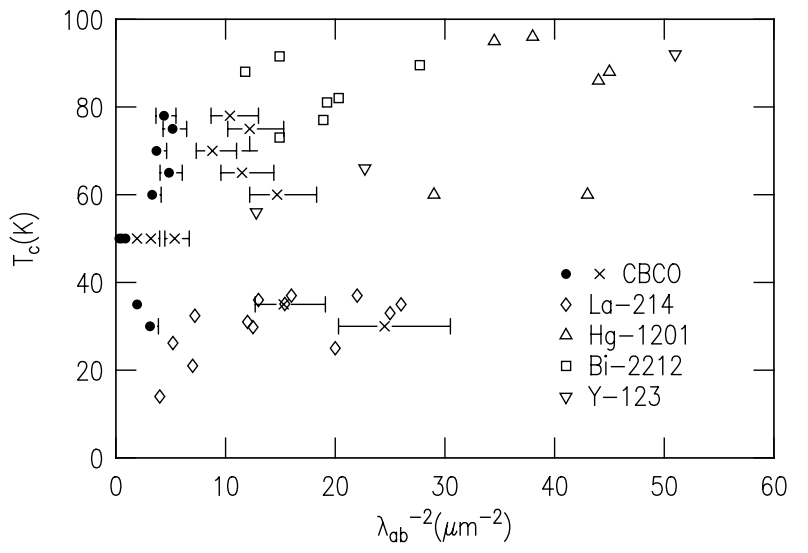

FIG. 3. Values of $T_{c}$ vs $\lambda_{a b}^{-2}$. The solid dots are the results for CBCO, using $\lambda_{a b, h}=\sqrt{d \Lambda / 2}$ (homogeneous distribution of superfluid density). The crosses are $\lambda_{a b, I L}=\sqrt{d_{I L} \Lambda / 2}$ (superfluid density localized in the IL layers). The open symbols are recent results for a number of bulk hole-doped cuprates: $\mathrm{La}_{2-x} \mathrm{Sr}_{x} \mathrm{CuO}_{4} \quad(\mathrm{La}-214) \quad[21] ; \mathrm{HgBa}_{2} \mathrm{CuO}_{4+\delta} \quad(\mathrm{Hg}-1201) ;$ $\mathrm{Bi}_{2} \mathrm{Sr}_{2} \mathrm{Ca}_{1-x} \mathrm{Y}_{x} \mathrm{Cu}_{2} \mathrm{O}_{8+\delta} \quad(\mathrm{Bi}-2212) \quad[22] ; \mathrm{YBa}_{2} \mathrm{Cu}_{3} \mathrm{O}_{7-\delta} \quad$ (Y123) [23]. 
densities for the CBCO samples are about a factor of 10 lower than for Y-123, although they have comparable $T_{c}$ 's,

It has been proposed that the superfluid screening in films could be supressed by proximity to the metalinsulator transition [27] or quantum fluctuations [28]. However the $2 \times 2$ superlattices have normal state resistances 10 times smaller than the metal-insulator critical resistance of $\sim 26 \mathrm{k} \Omega$ [11]. It appears that the penetration depths in these films are significantly larger than bulk cuprates with comparable $T_{c}$ 's. This may mean that these compounds are more efficient at producing high $T_{c}$ 's from a given superfluid density [29].

A clue to how this could come about comes from considering the layered structure of these films. If instead of assuming that the superfluid densities are homogeneously distributed, we assume instead that all of the superfluid density is localized in the IL layers, then $\lambda_{a b, I L}=\sqrt{d_{I L} \bar{\Lambda} / 2}$. In this case the calculated penetration depths (the crosses in Fig. 3) become comparable to the longest penetration depths reported for some cuprates: Although the average superfluid density in these films is low, the density in the IL layers might be higher, possibly promoting superconductivity at high temperatures.

We also note that the areal superfluid densities are about $2 \times 10^{14} \mathrm{~cm}^{-2}$ for the $5 / 2 / 5$ structures, making them ideal candidates for field effect experiments. The height of the surface barrier $E_{o}$ to formation of vortices is one of the crucial parameters to observe vortex quantum tunneling (VQT) [30]. $E_{o}$ is proportional to $\phi_{0}^{2} /\left(8 \pi^{2} \Lambda\right)$ and therefore inversely proportional to $\Lambda$ : the larger the Pearl length, the lower the barrier height.

In conclusion, we have investigated vortex matter in ultrathin $\left[\mathrm{Ba}_{0.9} \mathrm{Nd}_{0.1} \mathrm{CuO}_{2+x}\right]_{m} /\left[\mathrm{CaCuO}_{2}\right]_{n}$ systems using scanning SQUID magnetometry and susceptometry. We have given the first experimental evidence for Pearl vortices in the regime $d \ll \lambda_{L}$. This can be considered the closest attempt yet to investigate vortices in twodimensional systems (vortices of zero length). This experiment proves that extreme regimes (ultrathin films) are experimentally accessible through SSM and opens up several prospects of broad interest, especially if we consider that these topological defects may have analogies in other fields of physics. These measurements identify systems with very long penetration depths and relatively high $T_{c}$. This represents a further step to experimentally isolate the properties important for superconductivity in high- $T_{c}$ compounds. Finally, these systems potentially represent ideal systems to test novel theories and concepts for devices (VQT and field effect experiments).

This work has been partially supported by the ESF projects Pi-Shift, VORTEX, and QUACS. The authors would like to thank J. Blatter, J. Guikema, V.G. Kogan, V. Z. Kresin, and C.C. Tsuei for useful discussions.

[1] G. Blatter et al., Rev. Mod. Phys. 66, 1125 (1994).

[2] G. E. Volovik, Exotic Properties of Superfluid ${ }^{3} \mathrm{He}$ (World Scientific, Singapore, 1992).

[3] N. D. Mermin, in Quantum Fluids and Solids, edited by S. B. Trickey, E. Dwight Adams, and J.W. Duffy (Plenum, New York, 1977); D. J. Thouless, Topological Quantum Numbers in Nonrelativistic Physics (World Scientific, Singapore, 1998), and references therein.

[4] W. H. Zurek, Phys. Rep. 276, 177 (1996).

[5] T.W. B. Kibble, J. Phys. A 9, 1387 (1976).

[6] A. A. Abrikosov, Zh. Eksp. Teor. Fiz. 32, 1442 (1957) [Sov. Phys. JETP 5, 1174 (1957)].

[7] S. J. Bending, Adv. Phys. 48, 449 (1999)

[8] J. Pearl, Appl. Phys. Lett. 5, 65 (1964).

[9] V. G. Kogan, V.V. Dobrovitski, J. R. Clem, Y. Mawatari, and R. G. Mints, Phys. Rev. B 63, 144501 (2001).

[10] S. Scheidl, Phys. Rev. B 55, 457 (1997).

[11] G. Balestrino et al., Phys. Rev. B 62, 9835 (2000).

[12] G. Balestrino et al., Appl. Phys. Lett. 79, 99 (2001).

[13] J. R. Kirtley et al., Appl. Phys. Lett. 66, 1138 (1995).

[14] B.W. Gardner et al., Appl. Phys. Lett. 80, 1010 (2002).

[15] G. Balestrino, S. Lavanga, P. G. Medaglia, P. Orgiani, and A. Tebano, Phys. Rev. B 64, 020506 (2001).

[16] V. G. Kogan et al., Phys. Rev. B 63, 144501 (2001).

[17] S. -W. Han et al., Phys. Rev. B 62, 9784 (2000).

[18] Yu. V. Obukhov, J. Low Temp. Phys. 114, 277 (1999).

[19] V. G. Kogan, Phys. Rev. B 68, 104511 (2003).

[20] R. Mints, V. G. Kogan, and J. R. Clem, Phys. Rev. B 61, 1623 (2000).

[21] C. Panagopoulos et al., Phys. Rev. B 67, 220502 (2003).

[22] G. Villard, D. Pelloquin, and A. Maignan, Phys. Rev. B 58, 15231 (1998).

[23] C. Panagopoulos, J. R. Cooper, and T. Xiang, Phys. Rev. B 57, 13422 (1998).

[24] D. A. Bonn and W. N. Hardy, in Physical Properties of High Temperature Superconductors, edited by D. M. Ginsberg (World Scientific, Singapore, 1996),Vol. 5.

[25] P. G. de Gennes, Superconductivity of Metals and Alloys (Perseus, Reading, 1999).

[26] S. Wolf and V. Z. Kresin, Phys. Rev. B, 41, 4278 (1990).

[27] K. Semba and A. Matsuda, Phys. Rev. Lett. 86, 496 (2001).

[28] S. N. Artemenko and S. V. Remizov, Phys. Rev. Lett. 86, 708 (2001).

[29] Y. J. Uemura, in Proceedings of the International Workshop on Polarons and Bipolarons in HTS and Related Materials, edited by E. Salje, A. S. Alexandrov, and Y. Liang (Cambridge University Press, London, 1995), p. 453.

[30] L. I. Glazman and N. Ya. Fogel's, Sov. J. Low Temp. Phys. 10, 51 (1984). 